\title{
Idiopathic granulomatous mastitis: a new algorithm
}

\begin{abstract}
Idiopathic granulomatous mastitis (IGM) is a breast non-systemic and chronic inflammatory disease that mimics abscesses, mastitis or breast cancer of low incidence, with much controversy on its diagnosis and successful treatment. The aim of this study is to retrospectively associate in an integral manner, the clinical characteristics, and the radiologic findings in order to develop an algorithm to make diagnosis and treatment of this entity easy. A retrospective analysis was performed with 16 patients for whom histologically IGM diagnoses were confirmed and were treated in our center between January 2011 and December 2016. Clinical characteristics, radiological, and histological reports, as well as treatment regime and outcome were included in the analysis.
\end{abstract}

The clinical characteristics of the patients were very heterogeneous. The imaging studies performed were breast sonography and/or mammography, most of them with a BI-RADS classification of 4B (62\%), no MRI was conducted or deemed necessary. Histological specimens were obtained with core needle biopsies $(n=6)$ or excisional biopsy of the breast mass $(n=10)$. Patients were divided into three groups depending on the treatment they received; 1$)$ pharmacological-only group $(n=4)$ treated with antibiotic and prednisone; 2) surgical-only group stereotactically guided surgery $(n=6)$; or 3 ) pharmacological and surgical-group $(n=6)$ treated with pre-surgical antibiotic and pre and/or post-surgical prednisone. Recurrence occurred in two patients of the pharmacological-only group and in two patients of the pharmacological-surgery group, whereas there was no recurrence in the surgical-only group.

Idiopathic granulomatous mastitis is a breast-limited entity, with a chronic and generally benign course, with unknown etiology; but if not treated correctly there is a possibility of recurrence. Radiologic protocols are not sufficiently precise to establish a differential diagnosis, and while histopathological diagnosis is obligatory.

Clinical treatment guides must be developed for which we strongly recommend the following diagnosis and treatment algorithm for patients with granulomatous mastitis.

Keywords: idiopathic granulomatous mastitis, periductal mastitis, sarcoidosis, hormonal imbalance, breast mass
Volume 10 Issue 5 - 2019

\author{
Paola Iturralde-RosasPriego,' Javier \\ GomezPedroso-Rea,' Miguel Angel Mancera- \\ Resendiz,' Cecilia Ortiz-lturbide, ${ }^{2}$ Catalina \\ Romo-Aguirre, ${ }^{3}$ Daniela Stuht-Lopez,' Manuel \\ Ubiergo-García,' \\ 'Grupo de Alta Especialidad Ginecologica y Mamaria, Hospital \\ Ángeles del Pedregal, Mexico \\ ${ }^{2}$ Breast Imaging and Diagnosis, Hospital Angeles del Pedregal, \\ Mexico \\ ${ }^{3}$ Pathology Department, Hospital Ángeles del Pedregal, Mexico
}

Correspondence: Paola Iturralde-RosasPriego, MD, Grupo de Alta Especialidad Ginecologica y Mamaria, Hospital Ángeles del Pedregal, Camino a Santa Teresa \# 1055, Torre de Especialidades Quirurgicas Cons. 974, Col. Heroes de Padierna, Del. Magdalena Contreras, Ciudad de México, CP 10700, México, Tel +52(55)55684888 Email paola_iturralde@hotmail.com

Received:September 17, 2019 | Published: September 26, 2019
Abbreviations: IGM, idiopathic granulomatous mastitis; $\mathrm{H} \& \mathrm{E}$, haematoxylin-eosin; BI-RADS, breast imaging reporting and data system; MRI, magnetic resonance imaging

\section{Introduction}

Idiopathic granulomatous mastitis (IGM) is a rare benign chronic inflammatory breast disease of unknown etiology that may be clinically and radiologically mistaken with other diseases such as breast carcinoma, tuberculosis, fungal infections, periductal mastitis and sarcoidosis. ${ }^{1}$ IGM was originally described in 1972 by Kessler y Wolloch as breast masses characterized by florid granulomatous mastitis that were associated with granulomatous infections, trauma, or foreign body reaction (Kessler, 1972). ${ }^{2}$

Although no ethnic predisposition has been clearly documented for IGM, around of 1,400 cases have been worldwide reported until 2014, most of them found in Turkey, China, South Korea and United States. ${ }^{3}$ Particularly, Baslain et al reported that from a total of 1,106 women diagnosed with several benign breast pathologies in the Middle East (Saudi Arabia, Sudan, Jordan and Indonesia), IGM occurred in 20 of them $(1.6 \%){ }^{1}$
IGM is a chronic and localized disease, however local manifestations can mimic a malignant breast tumor. Although it is more common to have a unilateral clinical presentation, cases of bilateral IGM have been reported, occuring in any quadrant except subareolar region. ${ }^{4}$ The clinical presentation can be variable, however a firm palpable mass in the breast is the most common complaint, accompanied by a remarkable inflammation with presence or absence of pain. During the chronic period, several other potential complaints include nipple retraction, hyperemia of the breast skin, edema, ulceration, fistulae development and axillary lymphadenopathy. ${ }^{3}$

Even though diverse factors have been associated with IGM, the etiology has not been fully elucidated as well as the factor that triggers the endothelial damage. Various factors have been suggested to play a role in the etiology of the disease, such as autoimmune processes, hormonal imbalance (hyperprolactinemia), unknown microbiology agents, oral contraceptives, smoking, pregnancy, breast-feeding, and alpha 1-antitrypsin deficiency. One of the accepted hypothesis, suggest that an autoimmune process is responsible for this entity which could explain the positive impact of steroids as treatment, however this hypothesis has not been proven yet. ${ }^{1,5}$ 
The diagnosis of IGM can only be confirmed by histopathology, which is characterized by the presence of epithelioid and multinucleated giant cell granulomas limited to the mammary lobules accompanied by microabscesses, ${ }^{6}$ with epithelioid histiocytes, plasmocytes, lymphocytes, eosinophils, neutrophils and necrosis.? Also, the diagnosis can be made by the exclusion of inflammatory breast carcinoma and other infectious agents and non-infectious causes of granulomatous inflammation.

The most common mammographic appearance of the lesion is an asymmetrically increased density, which is not pathognomonic for this entity. Sonographic patterns of the disease are diverse and appear to be related to the histologic features. Findings include a masslike appearance, tubular/nodular hypoechoic structures and focal decreased parenchymal echogenicity with acoustic shadowing. 1,8

In cases where the etiology is defined, it is easy to follow treatment algorithms that include "limiting or removing the etiological factor", however in cases where these factor is unknown, the differential diagnosis and treatment can become more complicated. ${ }^{3}$ Even though algorithms have been proposed, ${ }^{7}$ IGM treatment remains controversial because of its great heterogeneity, thus there is no agreement about the most appropriated approach in these patients. ${ }^{4}$ However, the use of antibiotics, corticosteroids and surgery have been reported as recommended treatment options. ${ }^{69}$ Nevertheless, although it is considered a disease with benign course, in the $16-50 \%$ of the cases can relapse. ${ }^{6,9}$

Based on the low incidence and poor knowledge of IGM and its controversy on diagnosis and treatment, the aim of this study was to retrospectively associate in an integral way, the clinical characteristics and the radiologic findings in order to propose an algorithm for its diagnosis and treatment, facilitating the clinicians their practice. Our study features 16 women diagnosed with IGM in our institution between January 2013 and December 2017. The present study also aims to provide clinical evidence in order to help in the understanding of the different clinical forms in which IGM occurs and responds to the several conventionally established treatments.

\section{Patients and methods}

This retrospective study included sixteen women with IGM confirmed histopathologically who were treated at our center "Grupo de Alta Especialidad Ginecológica y Mamaria" between January 2013 and May 2017. Patient characteristics, symptoms, radiological and pathological results, as well as treatment and outcome results were analyzed retrospectively from medical records. Diagnosis of IGM was confirmed histopathologically by core needle biopsy of the breast lesions ( 6 cases) or from excisional biopsy of the breast mass (10 cases). All pathology slides were examined with haematoxylineosin (H\&E). Only one case was analyzed with special stains such as Ziehl-Neelsen for tuberculosis and Grocott Methenamine Silver for fungal infection based on histopathological findings and patient's laboral history.

The inclusion criteria were women of 18 years or more, with confirmatory histopathologic biopsy for IGM, and with a follow up in our center. The exclusion criteria were patients that denied to sign informed consent or that the biopsy gave another result different from IGM.

The criteria for selecting each patient's treatment was based on the size of the lesion, the severity of the symptoms, BIRADS classification, evolution and response to the pharmacological agents according to our own experience (Algorithm 1). Some patients underwent a single treatment with steroid agents (4 cases), surgery (6 cases) or a combination of surgery plus steroid agents (6 cases). The range of resolution of the cases with surgery varied from 7 days to 5 months.

Informed consent was obtained from all participants.

\section{Results}

The mean age of presentation was $39 \pm 10$ years, and the majority of our patients presented a unilateral affection, with predomination in the right breast characterized by a palpable mass. The most frequent radiological finding was microcalcifications categorized with a BIRADS 4, and only two cases were categorized as BIRADS 3. All of the cases had a histopathology study demonstrating chronic inflammation confirming the diagnosis of IGM, and half of the patients had ductal hyperplasia associated.

The patients were divided in three groups to receive treatment: pharmacological, surgery and combined treatment. There was recurrence in 2 patients $(12.5 \%)$, one from the pharmacological group and one from the combined treatment, whereas there was no recurrence in the surgery-only group, during a 32 month follow-up.

\section{Clinical characteristics}

Clinical features of the patients are shown in Table 1. The mean age of the patients was 39 10 years (range $25-53$ years). Twelve patients were premenopausal, 8 of them in reproductive age (only one was lactating), and 4 patients had surgical menopause. Lesions were observed in the left breast in 6 cases $(37.5 \%)$, in the right breast in 8 cases $(50 \%)$, and 2 cases $(12.5 \%)$ presented bilateral lesions. The size of the mass varied from $<1$ to $9 \mathrm{~cm}$ of diameter and among the cases reported, the lesions were widely distributed in the breasts, without any characteristic pattern.

None of the patients presented nipple inversion or retraction; only 4 cases had nipple discharge, whereas enlarged axillary lymph nodes were presented in 8 cases. Other known associated factors $^{3}$ such as oral contraceptives, smoking, hormonal imbalance (f.e. hyperprolactinemia), autoimmunity, and infections were also considered (Table 1), however its incidence was $25 \%$ or less.

\section{Radiologic studies}

Findings from imaging studies are presented in Table 2. All patients had a sonography examination, which was complemented with mammography in 10 of the cases, based on their age. Three cases were classified as BI-RADS 4, two cases as BI-RADS 4A and nine cases as BI-RADS $4 \mathrm{~B}$, while only two cases were reported as BIRADS 3.

Microcalcifications were found in the 10 women in which mammography was performed, however only in two of them the calcifications were of benign appearance whereas in the rest they resembled malignant lesions. We considered that in none of the cases the MRI was necessary (Table 2).

\section{Histological studies}

All cases had a histopathology report confirming the diagnosis of IGM (Table 3) with granulomas formation. In none of the cases malignancy was detected. Chronic inflammation data were observed 
in 14 patients $(87.5 \%)$, without available information of the other two cases. From the 14 subjects with chronic inflammation, in 10 of them acute inflammation was also reported (62.5\% from total). In half of the patients, focal xanthogranulomatous mastitis was described whereas in the other 8 patients was severe $(25 \%)$ or not specified $(25 \%)$. Foreign body-type mastitis was reported in one patient (12.5\%). Other associated histopathology findings include the presence of ductal hyperplasia in 8 patients $(50 \%)$, calcifications in 6 patients $(37.5 \%)$, fibrosis in 2 patients $(12.5 \%)$, necrosis in 4 patients $(25 \%)$ and micro abscesses in 2 patients (12.5\%) (Table 3).

\section{Treatment}

The clinical impression of each case in conjunction with the available studies at each moment dictated further diagnostic workup and therapeutic approach. Based on the treatment patients received, they were classified into three groups as follows: 1) the pharmacological-only group (4 cases, representing $25 \%$ ); 2 ) the surgical-only group (6 cases, representing 37.5\%); and 3 ) the pharmacological and surgical-group (6 cases, representing $37.5 \%$ ) (Table 4). Five of the patients (31.25\%) had previously received medical treatment in another center, without clinical improvement.

Pharmacological-only group: In these four patients (cases 1, 2, 9 and 10 (Figure 1)), the initial clinical diagnosis was abscess, one of them associated with inflammatory mastitis. Prior to our medical treatment, all of them were managed with oral antibiotic therapy, (dicloxacillin combined with levofloxacin or ceftriaxone). Once the core needle biopsy was performed in our center and a histological report was obtained, patients received prednisone for 6 weeks (case 2 previously received a 3 week-prednisone treatment) with weekly schemes of decreasing doses ( 15 to $5 \mathrm{mg} /$ day) due to a considerable reduction of the mass without resolution or recurrence. The treatment was combined with ceftriaxone ( $1 \mathrm{~g}$ daily for 3-5 days) and with lysine clonixinate or meloxicam/methocarbamol for pain control (Tables 2-4).

Surgical-only group: Excisional surgery were performed on six patients older than 40 years (cases 3, 4, 5, 13, 15 and 16), based on the mammography radiologic findings performed in their periodic gynecological examination, where the lesions found were classified as breast imaging reporting and data system (BI-RADS) 4 . In these cases, the excisional surgery was helpful as diagnosis and treatment, without recurrence (Tables 2-4).

Pharmacological and surgical-group: Six patients were included in this combined treatment scheme, all in reproductive age (cases 6 (Figure 2); case 7; and case 8 (Figure 3); cases 11, 12 and 14). The patient in case 8 , had the resolution of her pregnancy 4 months prior to the first symptoms; whereas the patient described in case 7 had a second trimester miscarriage. For both of them, the initial clinical diagnosis was an abscess. However, they did not receive any antibiotic or antiinflammatory treatment before being managed in our clinic. Only one of them (case 8) received a lactation inhibitor treatment (carbegoline). Based on the prior existence of the lesions that were being treated as mastitis, excisional surgery was the definitive treatment of choice, along with a previous antibiotic scheme and prednisone treatment before and/or after the surgery (Tables 1-4).

\section{Follow-up and outcome}

Median follow-up was of 32 months (range of 9-75). From the 16 patients that were included in this study, recurrence developed in 2 of the patients $(12.5 \%)$, one of the pharmacological-only group (case 2) and the other one of the pharmacological-surgery group (case 8), whereas there was no recurrence in the surgical-only group (Table 4).
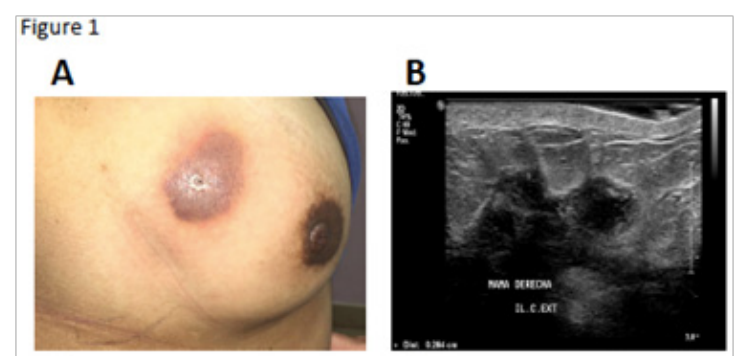

C

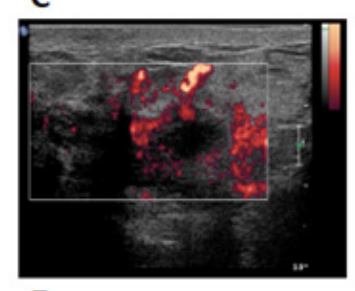

E

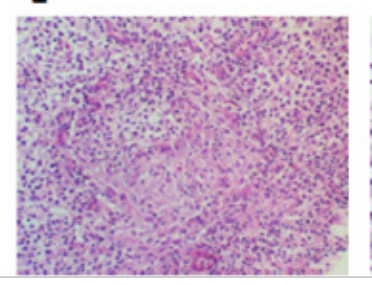

D

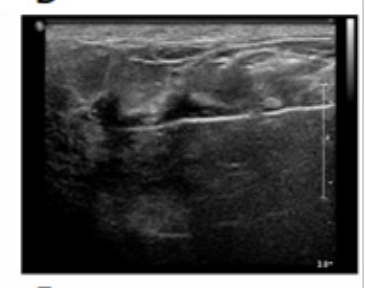

$\mathbf{F}$

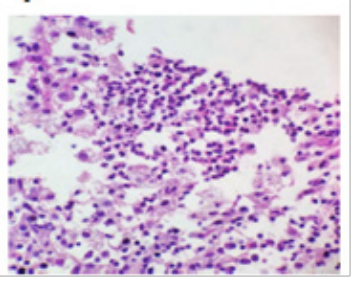

Figure I Clinical, radiologic and histopathology features of Case 2.

A. Clinical presentation of the lesion in the upper external quadrant on the right breast.

B. Breast sonography showing architectural distortion areas, heterogeneous mass with irregular edges, thickening of the skin with no lymphedema data.

C. Breast power Doppler sonography in which a significant vascularity is observed in the breast parenchyma.

D. Core needle biopsy of the lesion (caliber /4 G)

E. Breast histology stained with H\&E presenting multinucleated giant cells and inflammatory infiltrate composed of foamy macrophages, lymphocytes and few plasmocytes (IOX).

F. Breast histology stained with H\&E remarking inflammatory infiltrate of neutrophils surrounded by xanthomatous macrophages (40X).

Case 2 (pharmacological-only group) (Figure 1): Two weeks after the first scheme of pharmacological treatment $(10 \mathrm{mg}$ Prednisone every $12 \mathrm{~h}$ and 7.5/215 mg meloxicam/methocarbamol for 3 weeks and 10 days, respectively) erythema below the original lesion was documented considered as recurrence, thus antibiotic was included in the pharmacologic treatment and steroid management (prednisone) was continued (15 mg per day for 1 week, followed by $10 \mathrm{mg}$ per day in the second week). Afterwards, a satisfactory evaluation was observed, however the pharmacological treatment with prednisone was extended for 4 additional weeks (10 mg per day for 2 weeks, followed by $5 \mathrm{mg}$ per day in the second week). The patient is still under progress, and an excision of the lesion and the skin is under consideration. 


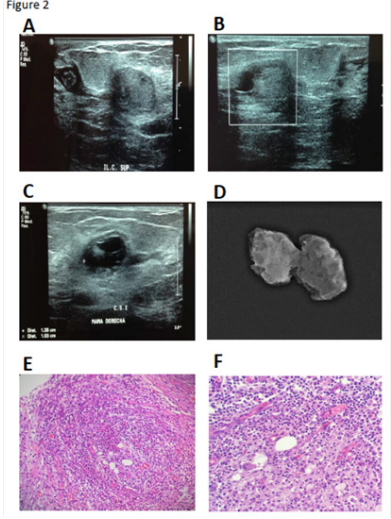

Figure 2 Radiologic and histopathology characteristics of Case 6.

A. Breast sonography showing a solid lesion with cystic component occupying most of the upper quadrants of the right breast with fistulization toward the skin.

B. Breast sonography posterior to the pharmacological treatment where fistula is resolved and vascularity decreases significantly.

C. Breast sonography after two months of pharmacological treatment denoting that the lesion tends to organize; the solid area begins to fade whereas the cystic area increases its volume.

D. Mammography image confirmed a total inclusion of the lesion in the mammary tissue extracted.

E. Breast histology stained with H\&E presenting inflammatory infiltrate composed of neutrophils, lymphocytes and foamy macrophages, diffusely disposed forming a xanthomatous reaction (IOX).

Breast histology stained with H\&E denoting IGM; multinucleated giant cells and inflammatory infiltrate composed of foamy macrophages, lymphocytes and few plasmocytes (40X).
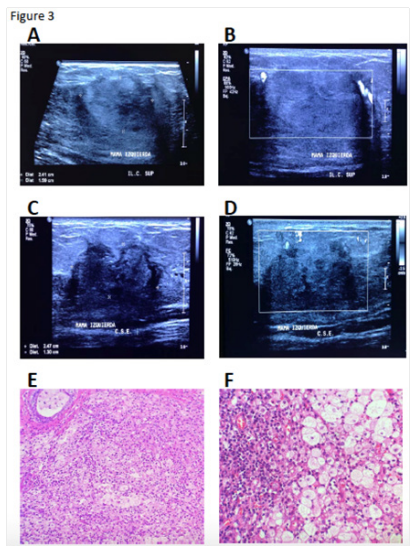

Figure 3 Radiologic and histopathology features of Case 8.

A and B: Initial breast sonography previous to pharmacological treatment, showing the irregular lesion with diffuse edges, heterogeneous echogenicity and peripheral vascularity, probably benign (BI-RADS 3).

C and D: Second breast sonography posterior to antibiotic treatment, where the lesion persists and is reclassified (BI-RADS 4A). Great irregularity is observed in contours with increased vascularity.

E and F: Breast histology stained with H\&E denoting chronic IGM (IOX and $40 \mathrm{X}$, respectively)

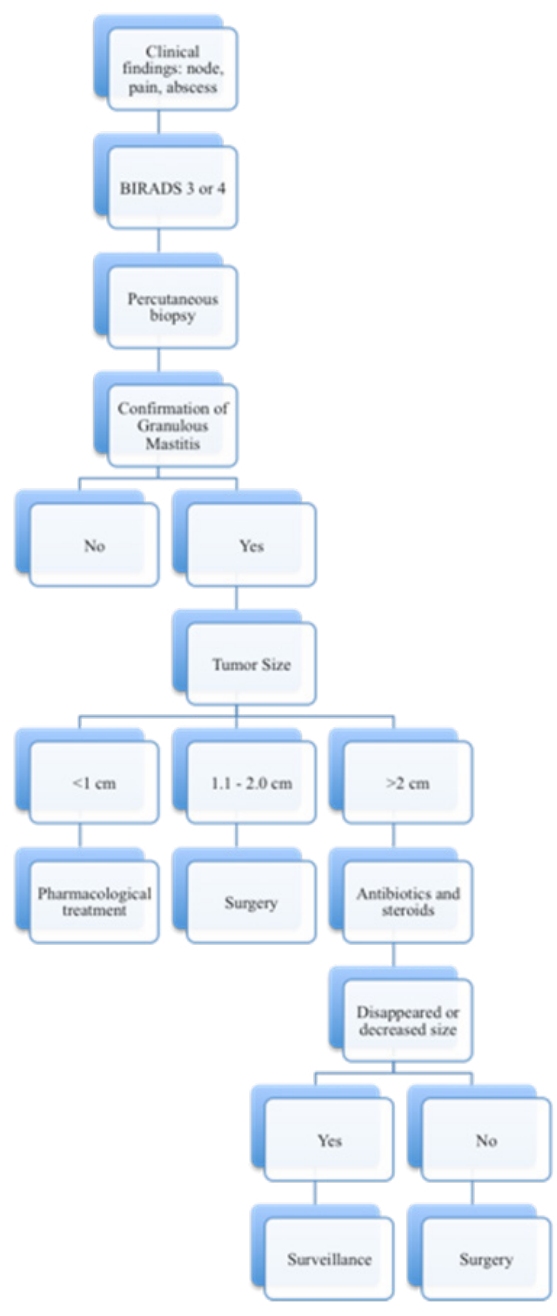

Figure 4 Proposed algorithm for diagnosis and treatment for xantogranulomatous mastitis.

Case 8 (pharmacological-surgery group) (Figure 3): After the second sonography the mass lesion was reclassified from BI-RDAS 3 to $4 \mathrm{~A}$ (Table 2 and Figure 3A-D). After the surgery, with a confirmed histopathology diagnosis of IGM, prednisone treatment was initiated (35 mg weekly for 2 weeks, $15 \mathrm{mg}$ weekly for 2 weeks and $10 \mathrm{mg}$ weekly for 2 weeks). A mass lesion $(1.9 \mathrm{x} 9 \mathrm{~cm})$ with irregular borders, with significant hydric content and with vascularity was identified by sonography in the same region of the breast, 12 weeks after the surgery (BI-RADS 3). Thus, prednisone treatment was initiated (10mg per day for a total of 5 weeks). At the end of this period, another breast sonography study reported that the lesion was heterogeneous, predominantly hypoechoic, irregular $(0.8 \times 1.3 \times 0.5)$ without vascularity (BI-RADS 2), adjacent to another identified lesion ( $7 \mathrm{~mm}$ ) with similar characteristics (Table 2). Five mg daily of prednisone was indicated for 1 month more. The last sonography reported a residual lesion with fibrotic scar formation. At present, the patient is under observation (Table 2-4).

\section{Discussion}

Idiopatic Granulomatous Mastitis (IGM) is a rare and benign, non-systemic breast disease that represents a small percentage of 
inflammatory pathologies, with an unknown etiology. There are no actual clinical guidelines for its diagnosis and/or treatment, ${ }^{4}$ because the majority of the available information on the disease is based on clinical trials. One of the most significant publications available, was made in 2010 by Kok and cols, ${ }^{6}$ and includes 43 patients, in a period between 1997-2009 in a Brunei Hospital. Generally, these patients need a prolonged and close follow-up because of the frequent recurrence in the disease.

In the present study, all of the patients were between the third and sixth decade of life, without any tendency findings regarding the pre or post-menopausal period. The usual clinical presentation involves a tumor or mammary abscess, which may involve skin and adjacent layers that might penetrate into pectoral muscles. Also, nipple discharge or retraction along with unilateral presentation and affection of lymph nodes are common in IGM. ${ }^{3}$ In our studied population, we found that the most frequent clinical presentation was breast tumor, along with pain and erythema, and contrary to the literature, none of our patients had nipple inversion and/or retraction. Kayahan and cols, ${ }^{10}$ published a series of cases that involved 31 patients, with breast tumors as the principal clinical manifestation, followed by pain and erythema, as our population did.

The diagnosis for this disease represents a challenge for the physician, as the breast sonography findings are non-specific. The typical appearance describes an irregular mass, heterogeneous, hypoechoic with posterior acoustic enhancement. On the other hand, mammography will describe a mass suspicious of malignancy, with heterogeneous composition, non-well defined borders that may even present spiculations. ${ }^{2,7,8}$ Recently, the use of magnetic resonance imaging (MRI) has been recommended as an additional resource in the diagnosis of this entity, which shows a homogeneous focal enhancement of a mass with irregular borders and distortion of the parenchima. ${ }^{11}$ In contrast, several publications, such as the one by Kayahan and cols ${ }^{10}$ and Kok and cols, ${ }^{6}$ described the insufficient diagnostic value that the radiologic studies have in order to differentiate the IGM from breast cancer, because of the radiologic similarity that both entities present although breast ultrasound has demonstrated to be a useful resource in patients with IGM accompanied by abscesses. In our population, 10 of the patients $(62.5 \%)$ presented malignancy characteristics during the radiologic assessment, but none of them were confirmed as malignant masses in the histopathological report, which goes accordingly with the world literature.

For an accurate diagnosis, it is necessary to have the histopathological report, in which specific findings must be found, such as lobar chronic inflammation with lymphocites, plasmocytes and non caseous granuloma. ${ }^{7,8,12}$

The correct treatment for IGM still remains controversial, as most of the world literature available is based on series of cases with poor significance. There are no actual guidelines for the management of the disease, therefore, the use of antibiotics, steroids and surgery have been the basis for treatment. ${ }^{12}$ In the majority of our population, surgery was performed, as it has been reported by several authors to have the least relapse, adverse effects and faster healing, when compared to medical treatment. ${ }^{13}$ Regarding the medical treatment, antibiotics should be reserved for those in which an infectious process is identified, however, as mentioned before, the diagnosis of this entity tends to be confusing. ${ }^{5,14}$

Steroids have demonstrated to be effective in reducing the size of the mass and even achieving complete remission, ${ }^{13}$ as we exemplify in our proposed treatment algorithm. DeHertogh and cols, ${ }^{15}$ among others, reported the use of steroids in this disease, becoming successful even in refractory cases. These authors used metilprednisone doses from 5 to $60 \mathrm{mg} /$ day, with gradual increases during the course of treatment and recommend a long term treatment with steroids that vary from one week to 22 months, although recidivant cases have been reported when patients abandon treatment. One of the main aspects to have in consideration is the adverse effect that steroids may produce, as it is a long-term treatment regime. ${ }^{5}$ The therapy with steroids is also recommended before surgery is performed, in order to achieve less invasive techniques and having best cosmetic results. Several other agents, such as methotrexate and azathioprine, have been recommended for reducing steroid adverse effects. Akblulut and cols, ${ }^{16}$ worked on a review of 84 published articles between 1972 and 2010, where 78 patients with IGM underwent steroid treatment. Nine of these patients left steroid based treatment because of recidivant masses and type 2 Diabetes Mellitus, for which methotrexate was initiated having a good response and therefore demonstrating the effectiveness of this agent for patients with IGM..$^{16,17}$

In Mexico, Peña and cols, ${ }^{18}$ published a series of cases in which for secondary reactions to the steroid treatment and recidivant disease, the addition of methotrexate to the established steroid regime, in a similar dose as the one used for rheumatoid arthritis, helped to achieve the remission of the disease.

In our study, we used prednisone $5 \mathrm{mg}$ /day prior to surgery, in $57.1 \%$ of the patients, in order to reduce the mass and achieve a better cosmetic result. Disease recurred in two patients, which represent $12.5 \%$ of our population, and is in accordance to the world literature, that reports a 10 to $50 \%$ recurrence rate. Nowadays, these patients continue in follow-up with steroid regime and will probably require surgery. ${ }^{9,19}$

Due to the low number of patients included in our study, it is difficult to obtain a significant statistical analysis. The rare incidence of this entity limits the number of cases that can be included in order to have a significant analysis, which is the main concern in the present study. We encourage clinicians to know this rare entity in order to have a clinical suspicion and follow the proposed algorithm for correct diagnosis and treatment (Figure 4). ${ }^{20-24}$

\section{Conclusion}

Idiopathic granulomatous mastitis is a breast-limited entity, with a chronic and benign course, with unknown etiology. Generally, it presents as a unilateral solid tumor, adhered to breast adjacent tissue and affecting the nipple morphology, for which it is most of the time confused with breast cancer as they both present similar clinical manifestations. Radiologic protocols are not precise to establish a differential diagnosis, and histopathological diagnosis is highly recommended.

The treatment remains controversial, as several regimes with steroids and antibiotics have been proposed, in addition to surgery excision of the lesion, which has demonstrated in the actual literature to be the best treatment, even though relapse of IGM is still frequent.

Due to the unusual entity, there is very few information on diagnosis and treatment for these group of patients, and continues to be not effective in all cases. Which is why we firmly believe that the algorithm proposed in this study based on our population may be very helpful for other clinicians to diagnose and treat patients with idiopathic granulomatous mastitis. 


\section{Acknowledgments}

None.

\section{Funding}

None.

\section{Conflicts of interest}

There is no conflict of interests in any of the authors.

\section{References}

1. Baslaim MM, Khayat HA, Al-Amoudi SA. Idiopathic granulomatous mastitis: a heterogeneous disease with variable clinical presentation. World J Surg. 2007;31(8):1677-1681.

2. Kocauglo M, Somuncu I, Ors F, et al. Imaging findings in idiopathic granulomatous mastitis. a review with emphasis on magnetic resonance imaging. J Comput Assist Tomogr. 2004;28(5):635-641.

3. Altintoprak F, Kivilcim T, Ozkan OV. Aetiology of idiopathic granulomatous mastitis. World J Clin Cases. 2014;2(12):852-858.

4. Akcan A, Akyildiz H, Deneme MA, et al. Granulomatous lobular mastitis: a complex diagnostic and therapeutic problem. World J Surg. 2006;30(8):1403-1409.

5. Sakurai K, Fujisaki S, Enomoto K, et al. Evaluation of follow-up strategies for corticosteroid therapy of idiopathic granulomatous mastitis Surg Today. 2011;41(3):333-337.

6. Kok KY, Telisinghe PU. Granulomatous mastitis: presentation, treatment and outcome in 43 patients. Surgeon. 2010;8(4):197-201.

7. Lacambra M, Thai TA, Lam CC, et al. Granulomatous mastitis: the histological differentials. J Clin Pathol. 2011;64(5):405-411.

8. Memis A, Bilgen I, Ustun EE, et al. Granulomatous mastitis: imaging findings with histopathologic correlation. Clin Radiol. 2002;57(11):10011006.

9. Joseph KA, Luu X, Mor A. Granulomatous mastitis: a New York public hospital experience. Ann Surg Oncol. 2014;21(13):4159-4163.

10. Kayahan M, Kadioglu H, Muslumanoglu M. Management of Patients with Granulomatous Mastitis: Analysis of 31 Cases. Breast Care (Basel). 2012;7(3):226-230.
11. Gunduz Y, Altintoprak F, Tatli Ayhan L, et al. Effect of topical steroid treatment on idiopathic granulomatous mastitis: clinical and radiologic evaluation. Breast J. 2014;20(6):586-591.

12. Skandarajah A, Marley L. Idiopathic granulomatous mastitis: a medical or surgical disease of the breast? ANZ J Surg. 2015;85(12):979-982.

13. Akcan $\mathrm{A}, \mathrm{Oz} \mathrm{AB}$, Dogan $\mathrm{S}$, et al. Idiopathic granulomatous mastitis: comparison of wide local excision with or without corticosteroid therapy. Breast Care (Basel). 2014;9(2):111-115.

14. Kiyak G, Dumlu EG, Kilinc I, et al. Management of idiopathic granulomatous mastitis: dilemmas in diagnosis and treatment. BMC Surg. 2014; $14: 66$.

15. DeHertogh D, Rossof A, Harris AA, et al. Prednisone management of granulomatous mastitis. N Engl J Med. 1980;303(14):799-800.

16. Akbulut S, Yilmaz D, Bakir S. Methotrexate in the management of idiopathic granulomatous mastitis: review of 108 published cases and report of four cases. Breast J. 2011;17(6):661-668.

17. Akbulut S, Arikanoglu Z, Senol A, et al. Is methotrexate an acceptable treatment in the management of idiopathic granulomatous mastitis? Arch Gynecol Obstet. 2011;284(5):1189-1195.

18. Peña G, Ruiz M. Idiopathic granulomatous mastitis treated with steroids and methotrexate. Ginecol Obstet Méx. 2011;79(6):373-376.

19. Atak T, Sagiroglu J, Eren T, et al. Strategies to treat idiopathic granulomatous mastitis: Retrospective analysis of 40 patients. Breast Dis 2015;35(1):19-24

20. Hwang MJ, Rogers A, Vidya R. Idiopathic granulomatous mastitis: rare but important. BMJ Case Rep. 2010.

21. Poovamma CU, Pais VA, Dolas SC, et al. Idiopathic granulomatous mastitis: a rare entity with a variable presentation. Breast Dis. 2014;34(3):101-104

22. Zaragoza Zaragoza C, Hostalet Robles F, Kosny P, et al. Idiopathic granulomatous mastitis: a condition with no definitive treatment. Cir Esp. 2013;91(9):615-616.

23. Ruiter AM, Vegting IL, Nanayakkara PW. Idiopathic granulomatous mastitis: a great imitator? BMJ Case Rep. 2010.

24. Pereira FA, Mudgil AV, Macias ES, et al. Idiopathic granulomatous lobular mastitis. Int J Dermatol. 2012;51(2):142-151. 